ACCEPTED MANUSCRIPT

\title{
Live cell superresolution-SIM imaging analysis of the intercellular transport of microvesicles and costimulatory proteins via nanotubes between immune cells
}

To cite this article before publication: Henriett Halasz et al 2018 Methods Appl. Fluoresc. in press https://doi.org/10.1088/2050-6120/aad57d

\section{Manuscript version: Accepted Manuscript}

Accepted Manuscript is "the version of the article accepted for publication including all changes made as a result of the peer review process, and which may also include the addition to the article by IOP Publishing of a header, an article ID, a cover sheet and/or an 'Accepted

Manuscript' watermark, but excluding any other editing, typesetting or other changes made by IOP Publishing and/or its licensors"

This Accepted Manuscript is () 2018 IOP Publishing Ltd.

During the embargo period (the 12 month period from the publication of the Version of Record of this article), the Accepted Manuscript is fully protected by copyright and cannot be reused or reposted elsewhere.

As the Version of Record of this article is going to be / has been published on a subscription basis, this Accepted Manuscript is available for reuse under a CC BY-NC-ND 3.0 licence after the 12 month embargo period.

After the embargo period, everyone is permitted to use copy and redistribute this article for non-commercial purposes only, provided that they adhere to all the terms of the licence https://creativecommons.org/licences/by-nc-nd/3.0

Although reasonable endeavours have been taken to obtain all necessary permissions from third parties to include their copyrighted content within this article, their full citation and copyright line may not be present in this Accepted Manuscript version. Before using any content from this article, please refer to the Version of Record on IOPscience once published for full citation and copyright details, as permissions will likely be required. All third party content is fully copyright protected, unless specifically stated otherwise in the figure caption in the Version of Record.

View the article online for updates and enhancements. 


\section{Live cell superresolution-SIM imaging analysis of the intercellular transport of microvesicles and costimulatory proteins via nanotubes between immune cells}

Halász, Henriett ${ }^{1,+}$, Ghadaksaz, Ali Reza ${ }^{1,2,+}$, Madarász, Tamás ${ }^{1}$, Huber, Krisztina ${ }^{2}$, Harami, Gábor ${ }^{3}$, Tóth, Eszter Angéla ${ }^{2}$, Osteikoetxea-Molnár, Anikó ${ }^{2}$, Kovács, Mihály $^{3}$, Balogi, Zsolt ${ }^{5}$, Nyitrai, Miklós ${ }^{1,4}$, Matkó, János ${ }^{2, *}$, Szabó-Meleg, Edina $^{1,4, *}$

${ }^{1}$ Department of Biophysics, Medical School, University of Pécs, Szigeti street 12, H-7624, Pécs, Hungary

${ }^{2}$ Department of Immunology, and ${ }^{3}$ Department of Biochemistry, Faculty of Science, Eötvös Loránd University, Pázmány Péter street 1/C, H-1117 Budapest, Hungary

${ }^{4}$ Szentágothai Research Center, University of Pécs, Ifjúság street 20, H-7624, Pécs, Hungary

${ }^{5}$ Department of Biochemistry \& Medical Chemistry, University of Pecs, Medical School

H-7624 Szigeti str. 12, Pecs, Hungary

*Corresponding authors: Janos Matko PhD, DSc (matko@elte.hu) and Szabo-Meleg Edina, $\mathrm{PhD}$ (edina.meleg@aok.pte.hu )

+ The authors equally contributed to the project

Keywords: membrane nanotubes, SR-SIM imaging, microvesicle transport, microtubule, actin filaments, motor proteins, lymphocytes, macrophages

Running title: intercellular transport of microvesicles and CD86 via nanotubes assessed by superresolution microscopy 
ABSTRACT:

Membrane nanotubes are transient long-distance connections between cells that can facilitate intercellular communication. These tethers can form spontaneously between many cell types, including cells of the immune and nervous systems. Traffic of viral proteins, vesicles, calcium ions, mRNA, miRNA, mitochondria, lysosomes and membrane proteins/raft domains have all been reported so far via the open ended tunneling nanotubes (TNTs). Recently we reported on existence of plasma membrane derived $\mathrm{GM}_{1} / \mathrm{GM}_{3}$ ganglioside enriched microvesicles and costimulatory proteins in nanotubes connecting B lymphocytes, the way they are formed and transported across TNTs, however, still remained unclear. Here, using live cell confocal and Structured Illumination (SR-SIM) superresolution imaging, we show that B cells respond to bacterial (Cholera) toxin challenge by their subsequent internalization followed by rapid formation of intracellular microvesicles $(M V s)$. These MVs are then transported between adjacent B cells via nanotubes. Selective transport-inhibition analysis of two abundant motor proteins in these cell types demonstrated that actin-based non-muscle myosin 2A dominantly mediates intercellular MV-transport via TNTs, in contrast to the microtubule-based dynein, as shown by the unchanged transport after inhibition of the latter. As suggested by SR-SIM images of GFP-CD86 transfected macrophages, these costimulatory molecules may be transferred by unusually shaped MVs through thick TNTs connecting macrophages. In contrast, in B cell cultures the same GFP-CD86 is dominantly transported along the membrane wall of TNTs. Such intercellular molecule-exchange can consequently improve the efficiency of antigen-dependent $\mathrm{T}$ cell activation, especially in macrophages with weak costimulator expression and $\mathrm{T}$ cell activation capacity. Such improved $\mathrm{T}$ cell activating potential of these two cell types may result in a more efficient cellular immune response and formation of immunological memory. The results also highlight the power of superresolution microscopy to uncover so far hidden structural details of biological processes, such as microvesicle formation and transport. 


\section{INTRODUCTION}

Membrane nanotubes (NTs), thin and long cellular protrusion structures connecting cells, were first described more than a decade ago (Onfelt, Nedvetzki et al. 2004, Rustom, Saffrich et al. 2004). Since then, a number of interesting functional capabilities of these nanostructures have been described, such as intercellular signaling $/ \mathrm{Ca}^{2+}$ transfer and transport of protein molecules, prions, vesicles, membrane cargos, nucleic acids or even organelles (Davis and Sowinski 2008, Gerdes and Carvalho 2008, Gurke, Barroso et al. 2008, Sherer and Mothes 2008, Davis 2009).

NTs show high diversity in length, thickness and structure (Austefjord et al. 2014). Concerning their thickness, they are usually characterized as ,thin" ( 100-300 nm diameter) or 'thick' ( 400-1000 nm diameter) nanotubes. In vitro they always contain actin filaments (Factin), and the thick NTs often contain microtubules, as well.

Smaller entities, like prions or viruses were reported to be transported across any types of NTs, while transport of larger vesicles and organelles or 'surfing' of bacteria were reported mainly through the surface or interior of the thick, open-ended, so called 'tunneling nanotubes' (TNTs) (Onfelt, Nedvetzki et al. 2006, Gerdes, Bukoreshtliev et al. 2007, Osteikoetxea-Molnar, Szabo-Meleg et al. 2016). After exploring membrane nanotubes, cells of the nervous and immune systems were studied most deeply, including among others macrophages, neurons, astrocytes, T cells, NK cells and their targets. In addition, functional significance of nanotubes in bridging tumor cells has been nicely demonstrated recently and now it represents a new research line with continuously increasing high medical interest (Lou, Fujisawa et al. 2012, Osswald, Jung et al. 2015, Ady, Thayanithy et al. 2016, Lou 2016).

We have shown recently that mature B lymphocytes also contain microtubules in their thick, tunneling nanotubes, which are able to transport organelles, such as mitochondria or lysosomes, as well (Osteikoetxea-Molnar, Szabo-Meleg et al. 2016). Critical importance of membrane lipids (among others of gangliosides/glycosphingolipids or cholesterol) in NTformation were also reported recently (Delage and Zurzolo 2013, Toth, Oszvald et al. 2017). In spite of this continuously accumulating knowledge on membrane nanotubes, many questions about the mechanism and regulation of their formation, the direction of their growth, as well as their functional significance in viyo (Seyed-Razavi et al. 2013, Caneparo et al. 2011; Osswald et al. 2015; Rehberg et al. 2016; Saenz-de-Santa-Maria et al. 2017; Teddy and Kulesa 2004; Vignais et al. 2017) still remained open, although the latter publications firmly support that the nanotubes may exist and function in various in vivo environments.

In the present study we focused on revealing the intercellular transport properties of B cells' and macrophages' nanotubes, as a continuation of our recent work on basic mechanistic properties of B cell nanotube formation (Osteikoetxea-Molnar, Szabo-Meleg et al. 2016, Toth, Oszvald et al. 2017). Here we investigated the formation and transport of intracellular (IC) microvesicles (MVs) after challenging immature (unable to form nanotubular bridges) or mature (with high capacity to form nanotubular networks) B lymphocyte cells with bacterial toxin, the Cholera toxin B subunit (CTX-B) binding with high selectivity/affinity to $\mathrm{GM}_{1} / \mathrm{GM}_{3}$ gangliosides at the cell surface. This process tried to model an in vivo often appearing event, the interaction of bacterial toxins with $\mathrm{B}$ cell surface.

Using Alexa488-CTX-B and time-lapse live cell confocal (CLSM) and SR-SIM imaging we intended to understand how the ganglioside/CTX-B-enriched microvesicles (MV) (or multivesicular bodies; MVB) are formed and how are they transported through the tunneling nanotubes connecting B cells. We also investigated how the membrane ganglioside level correlates with formation of such intracellular MVs. Particularly, we were also interested in the mechanism of their transport, namely what type of motors proteins are involved in this process. 
CD86 is one of the essential B7-family costimulatory proteins expressed on various antigen presenting cells (such as dendritic cells, B cells or macrophages) that play a key role in amplifying TCR-dependent T cell activation/cellular immune response, independently of the quantitative aspects of TCR-antigen recognition. Therefore, CD86 may critically set the magnitude of $\mathrm{T}$-cell mediated cellular immune response through providing an additional 'second signal' upon binding to their CD28 receptors on T cells (Janakiram, Abadi et al. 2012).

Monitoring intercellular trafficking of GFP-CD86 in transfected B cells and macrophages, utilizing advantages of SR-SIM imaging (Vangindertael, Camacho et al. 2018) revealed that this essential costimulatory molecule can be exchanged among both cell types. In B cells it is transported mostly in the membrane wall of nanotubes, while in macrophages dominantly via large, elongated 'microvesicle-like' structures, inside the TNTs. Clarification of the nature of such mobile intracellular CD86 compartments, however, needs further detailed investigations. Our results, also demonstrating power of superresolution microscopy (Vangindertael, Camacho et al. 2018) over confocal microscopy to reveal structural details of biological processes, such as microvesicle transport, shed light on some new mechanistic details of the nanotube-mediated cellular communication between cells of the immune system.

\section{EXPERIMENTAL DETAILS}

\subsection{Cells, culture and labeling.}

$\mathrm{B}$ cells of murine origin with two different stages of maturation/differentiation were used in this study, A20 mature B cells (ATCC TIB208, I-Ad/Ed+) and 38C13 immature B cells (Caspi, Taya et al. 1995). These murine B and macrophage (RAW 264.7; ATCC $^{\circledR}$ TIB-71 $^{\mathrm{TM}}$ ) cell lines, were cultured in RPMI-1640 medium, supplemented with $2 \mathrm{mM}$ L-glutamine, $1 \mathrm{mM} \mathrm{Na}$ pyruvate, $50 \mu \mathrm{M}$ 2-mercaptoethanol, antibiotics and $10 \% \mathrm{FCS}$, and were kept at $37^{\circ} \mathrm{C}$ in $5 \%$ $\mathrm{CO}_{2}$ incubator. Expression of cell markers during tissue culture were continuously checked using immunocytochemical labeling and BD FACSAria III flow cytometer (BD, San Jose, CA, USA).

\subsection{Reagents, cell transfection \& treatments}

Fibronectin (Sigma-Aldrich, St. Louis, MO, USA) was used as optimal ECM support (Osteikoetxea-Molnar, Szabo-Meleg et al. 2016) for studying B cells in live cell imaging chamber.

Alexa488/Alexa647-Cholera toxin B were purchased from Life Technologies (Carlsbad, USA). Ciliobrevin D, specific inhibitor of dynein motor proteins were from Sigma-Aldrich. P-nitroblebbistatin, inhibitor of non-muscle myosin 2A (Kepiro, Varkuti et al. 2014) was purchased from Optopharma Ltd (Hungary). For live cell CD86 experiments, RAW246.7 murine macrophages and A20 murine B lymphocytes were electroporated by Amaxa Nucleofector IIb device (programs T-020 and L-013 were used, respectively) according to the manufacturer's instruction. Briefly, for each sample the mixture of $87 \mu \mathrm{l}$ of nucleofector solution and $19 \mu \mathrm{l}$ of supplement was prepared and added to the cells together with $2 \mu 1$ GFP-CD86 DNA construct (pCMV6-AC-GFP; OriGene Technologies, Rockville, MD, USA). The transfected cells were then passaged into a fibronectin coated glass petri dish and incubated overnight $\left(37^{\circ} \mathrm{C}, 5 \%\right.$ CO2). A pCMV6-AC-GFP vector with no insert was used as control in the experiments. To visualize endogenous CD86 cells were fixed with 4\% paraformaldehyde (PFA) for 10 min, at room temperature, then permeabilized with $0.1 \%$ Triton X-100+5\% BSA (Sigma-Aldrich, St. Louis, MO, USA) for $20 \mathrm{~min}$ at RT and incubated with CD86 (B7-2) monoclonal antibody (GL1, BD Biosciences, Franklin Lakes, NJ, USA) for $1 \mathrm{~h}$ at RT. After washing step the cells 
were incubated with donkey anti-rat biotinylated secondary antibody (Jackson ImmunoResearch Europe Ltd. UK) for $24 \mathrm{hrs}$ at $4^{\circ} \mathrm{C}$ in $0.05 \%$ thimerosal (Sigma-Aldrich, St. Louis, MO, USA) containing blocking solution then after $4 \mathrm{hrs}$ incubation with the ABC complex (Vectastain ${ }^{\circledR}$ Elite ${ }^{\circledR}$ ABC-HRP Kit, Vector Laboratories, Burlingame, USA) the signal was amplified by Alexa594-conjugated tyramide (Thermo Fisher Scientific, Waltham, MA, USA) and $\mathrm{H}_{2} \mathrm{O}_{2}$ (Sigma-Aldrich, St. Louis, MO, USA) for $10 \mathrm{~min}$ at RT, finally cells were mounted in Vectashield (Vector Laboratories, Burlingame, CA, USA) medium. To visualize Caveolin-1 RAW 264.7 macrophages were first labelled with anti-Cav-1 monoclonal antibody for 30 min at RT, then goat anti-mouse Alexa555 was used as secondary antibody after the previously mentioned fixation and permeabilization procedures (see above). AntiCav-1 primary and anti-mouse Alexa555 secondary antibodies were the kind gift of Prof. Anna L. Kiss (Semmelweis University, Faculty of Medicine, Department of Anatomy, Histology and Embryology, Budapest, Hungary).

\subsection{Quantitative measurement of NT growth and vesicle transport with live cell imaging}

Borosilicate bottomed chambers (P35G-0.170-14-C MatTek In Vitro Life Science Laboratories, Bratislava, Slovak Republic) were coated (or not) with $10 \mu \mathrm{g} / \mathrm{ml}$ fibronectin overnight to model physiological circumstances; temperature was controlled at $37 \pm 0.1{ }^{\circ} \mathrm{C}, \mathrm{CO}_{2}$ was controlled at $5 \% .10^{6}$ cells/well were incubated at these conditions for 1 hour. The number of NT-growing cells were counted in each fields, while the fluorescently labelled microvesicles were counted in the nanotubes, as mobile ones or not, depending on their average displacement obtained from their motion trajectories, using SR-SIM imaging and Zeiss ZEN software. Images were analysed with FIJI software (NIH, Bethesda, MD, USA), for velocity calculation the MultipleKymograph plugin of FIJI

(https://www.embl.de/eamnet/html/body_kymograph.html) was used. ImageJ/TrackMate plugin was used to identify and then track vesicle movement between cells via TNTs.

\subsection{Microscopic Imaging}

Nanotube formation was visualized with Zeiss LSM 710 Confocal Laser Scanning Microscope (CLSM) at 63x magnification (oil immersion objective; N.A.: 1.4). For imaging nanotube growth, the cells were labelled with Alexa488 or Alexa 647 conjugated CTX-B membrane ganglioside-specific probes at $40 \mu \mathrm{g} / \mathrm{ml}$ concentration (cells were incubated on ice for $20 \mathrm{~min}$, in the presence of fluorescently labelled CTX). Cells were then washed once with PBS buffer and once with RPMI+10\% FCS+mercaptoethanol medium and placed on the $10 \mu \mathrm{g} / \mathrm{ml}$ fibronectin coated (overnight) microchambers. Records were taken and analyzed with the Zen black software and further analyzed using Image J (FIJI; Wayne Rashband, NIH, Washington, USA) and occasionally Imaris 8.2 (Bitplane, Zürich, Switzerland) softwares. Superresolution (dlat: 80-90 nm) SR-SIM imaging was performed in a Zeiss Elyra S1 (63x; N.A.:1.4 objective) microscope system with live cell imaging conditions. Images were acquired with five grid rotations and analyzed with the ZEN (Black or Blue versions) software.

\subsection{Statistical analysis}

Occurrence frequencies were calculated from at least 20 images/sample ( $\sim 40-50$ cells/field; $\tau 800$ cells/sample) and given as mean \pm SD. The frequency of nanotube forming cells were calculated as a ratio of cells growing at least one nanotube per all cells visible in the particular field. The number of mobile vesicles/nanotube value was determined on the basis of their motion trajectories recorded in a 10 min interval from at least three independent cell samples (ca. $50 \mathrm{NTs}$ /sample). The image analysis and the statistical calculations, including significance 
tests (using Student t-test), were made with ImageJ/FIJI and SigmaPlot 10.0 or Origin 8.0 statistical softwares, respectively.

\subsection{Flow cytometry and trypan blue quenching experiments}

A20 lymphocytes' pellets were incubated with $40 \mu \mathrm{g} / \mathrm{ml}$ Alexa488-conjugated CTX-B on ice for 20 min Control samples were kept on ice without dyes added. Samples were washed twice at $4^{\circ} \mathrm{C}$, then pellets were re-suspended in pre-warmed PBS and incubated at $37^{\circ} \mathrm{C}$ for 2 min or $30 \mathrm{~min}$ to allow and facilitate CTX-B internalization. Samples were then placed on ice and immediately analyzed for green fluorescence (BPF 530/30) by a BD FACS Canto II flow cytometer (BD, San Jose, CA, USA). Cell debris and dead cells were excluded from the analysis based on FSC (forward scatter) vs. SSC (side scatter) dot plot and gating. Extracellular Alexa488-CTX signal was quenched by adding $0.04 \%$ of cell impermeable dye trypan-blue (Thermo Fisher Scientific, Waltham, MA, USA), hence fluorescence intensities of the remeasured samples were representative of internalized signal. Data/were analyzed by FACS DIVA 6.0 software (BD, San Jose, CA, USA), and displayed as relative mean of fluorescence normalized to unstained control cells.

For live cell SR-SIM imaging A20 B lymphocytes were treated with $0.1 \%$ trypan-blue dye for 2 minutes after Alexa488-CTX labeling (see above). To yisualize the quenching effect of trypan-blue on Alexa dye labeling CD86, A20 lymphocytes were first labelled with anti-CD86 monoclonal antibody (GL1, BD Biosciences, Franklin Lakes, USA) for $30 \mathrm{~min}$ at RT, then goat anti-rat Alexa488 (Thermo Fisher Scientific, Waltham, MA, USA) was used as secondary antibody. The cells were washed twice and Trypan-blue was added in $0.1 \%$ concentration to quench the fluorescence of the Alexa488 dye.

\section{RESULTS}

\subsection{Challenging B cells' plasma membrane with Cholera toxin B results in rapid formation of intracellular microvesicles detected by SR-SIM}

Since immature (38C13) and mature (A20) murine B cells are substantially different in their capacity to form nanotubes (Osteikoetxea-Molnar, Szabo-Meleg et al. 2016), first we compared here their response to bacterial toxin. Cells were incubated with Alexa488-Cholera toxin $\mathrm{B}$ subunit known to bind to $\mathrm{GM}_{1} / \mathrm{GM}_{3}$ gangliosides in the plasma membrane of cells (similarly to various enterotoxins). $30 \mathrm{~min}$ after addition, small, spherical microvesicles (MVs) could be detected in both cell types, although in largely different extent, under live cell imaging conditions, at $37^{\circ} \mathrm{C}$ and $5 \% \mathrm{CO}_{2}$ (Fig. 1A-D), using SR-SIM imaging. Notice that immature $\mathrm{B}$ cells (38C13) formed much less ganglioside-rich vesicles (and more slowly) than the mature B cells (A20) did (Fig. 1E). Concerning the ganglioside-rich microvesicles, we found that they form in the cytoplasm rapidly after internalization (formation saturates in ca. $30 \mathrm{~min}$ ) (Fig. 1E) and are very heterogenous in size, in a range of $\sim 200-1000 \mathrm{~nm}$ (see e.g. Fig. 3D).

We should note here that the lateral resolution of CLSM (Schermelleh, Heintzmann et al. 2010) was not sufficient enough to resolve clearly these microvesicles and determine their size- or velocity-distribution. SR-SIM imaging, however, with $\mathrm{d}_{\text {lat: }} \sim 80-90 \mathrm{~nm}$ provided a sufficient lateral (x-y) resolution to do that (see: Supplementary Fig. 1). 

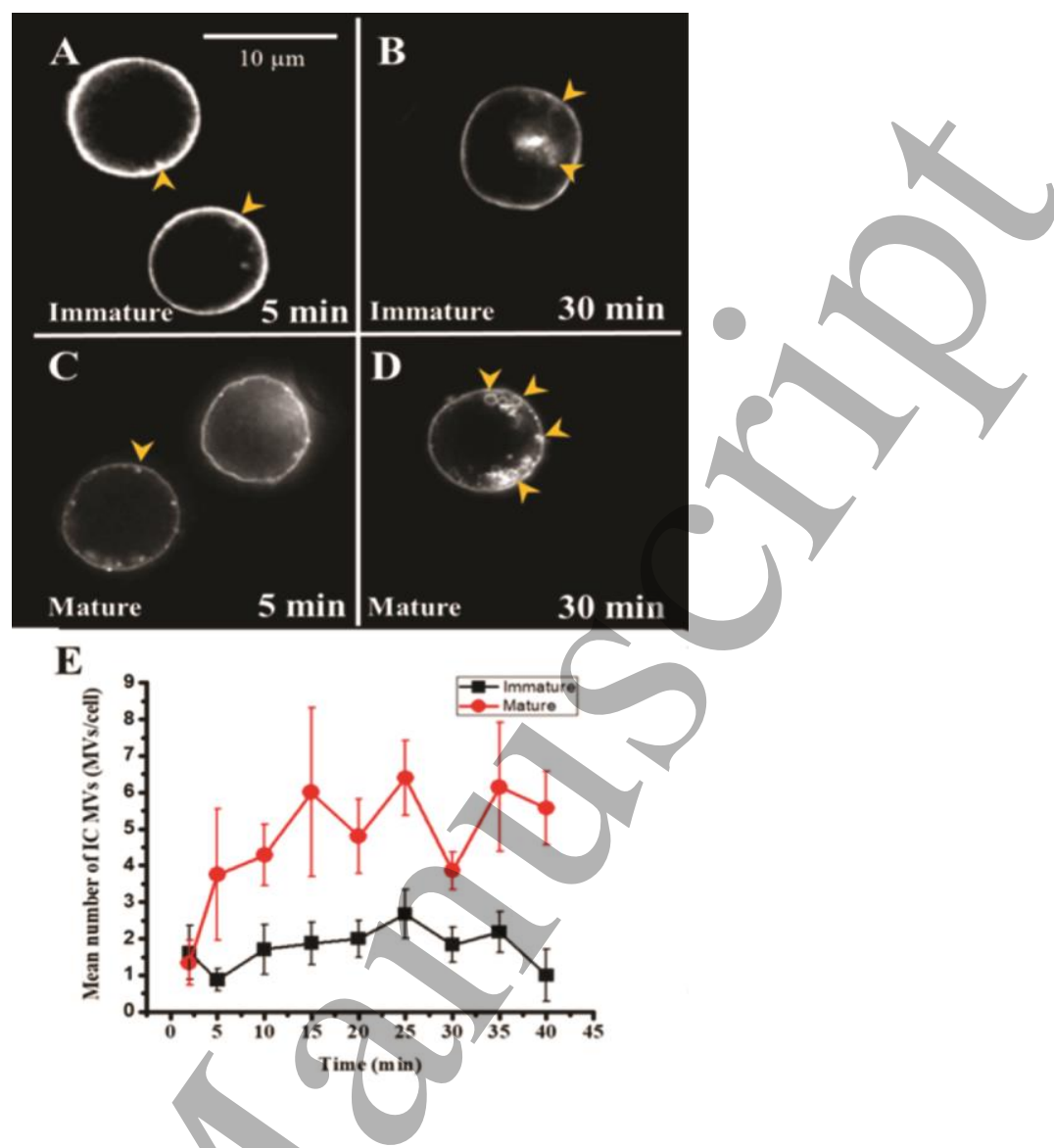

Figure 1. Cholera toxin $B$ challenge of $B$ cells results in formation of intracellular microvesicles. Representative SR-SIM images of 38C13 immature (A,B) and A20 mature (C,D) murine B cells are shown 5 and 30 min after CTX-B stimulation, respectively. The fluorescence originates from membrane bound Alexa488-CTX-B molecules. The quantitative difference between MV formation in immature and mature B cells, respectively, is shown in panel $\mathbf{E}$, where the mean number of intracellular vesicles/cell is displayed in time. Data at each time point are displayed as mean +/- SD of 3-4 independent experiments (including ca. 30-50 cells/sample).

\subsection{Characterization of the ganglioside-rich MVs of B cells}

The fact that the intercellular conduits connecting $B$ lymphocytes contain $\mathrm{GM}_{1} / \mathrm{GM}_{3}$ ganglioside-rich microvesicles has already been reported by us, recently (Osteikoetxea-Molnar, Szabo-Meleg et al. 2016). While confocal microscopy with its $d_{\text {lat: }} 240 \mathrm{~nm}$ resolution could not resolve smaller individual microvesicles formed and transported inside and between cells, SR-SIM imaging could nicely overcome this limitation (Supplementary Fig.1). The improved resolution is remarkably visible at the 'growth cone'-like structure of the TNTs at the interfaces with the cell membranes of the two adjacent B lymphocytes. Here the CLSM can show only a patchy and blurry fluorescence picture, while SR-SIM draws sharply contrasted (fluorescent), membrane covered, small globular vesicle structures. This means that applying live cell SRSIM imaging, there is a good chance to monitor the mechanistic details of the intercellular MV-transport.

Upon challenging B cells with Alexa488-Cholera-toxin B $(40 \mu \mathrm{g} / \mathrm{ml})$ we observed formation of small intracellular vesicles shown in Figure 1. Internalization of this fluorescent ligand bound to their cell surface $\mathrm{GM}_{1} / \mathrm{GM}_{3}$ ganglioside receptors was monitored quantitatively by flow cytometry (Fig. 2A-D) and also demonstrated by SR-SIM microscopy 
(Fig. 3A,B). Trypan-blue quenching of surface bound (extracellular) fluorescence could sensitively discriminate it from the intracellular fluorescence with robust statistical value in flow cytometry (Patino, Soriano et al. 2015). B cells gated by the forward vs. side scatter diagram (Fig. 2A) show significantly higher fluorescence in the absence (Fig. 2B) than in the presence of trypan-blue (TB) (Fig. 2C and D) at both 2 and 30 minutes after CTX administration. At shorter times ( $2 \mathrm{~min}$ ) however, the extent of the quenching was much higher than that of at longer times $(30 \mathrm{~min}$ ) (Fig. 2D). This clearly demonstrates that the internalization is continuously running and saturates in ca. 30 minutes after addition of the bacterial toxin. These observations are further confirmed by the SR-SIM images showing similar effects of trypan-blue quenching (Fig. 3A,B). These representative images demonstrate that while the intracellular, internalized vesicle fluorescence does not change upon TB addition, the fluorescence at the plasma membrane got highly reduced (see yellow arrowheads).

Fig. 3C shows a representative image of mature A20 B cells $30 \mathrm{~min}$ after CTX-B challenge, where IC MVs already appeared. These cells were re-stained with Alexa647-CTX$\mathrm{B}$ at this time (middle image). A merge of the two images (right), demonstrates coexistence of plasma membrane and intracellularly localized ganglioside-CTX-B complexes/MVs in the same cell. As shown by a representative line scan diagram (Fig. 3D), the majority of these intracellular microvesicles have 200-500 nm diameter. Some larger (ca. 2-4 times as large) vesicular structures could also be observed (see also in (Osteikoetxea-Molnar, Szabo-Meleg et al. 2016). These are supposed to be multivesicular bodies (MVBs), appearing in a small number. Their detailed characterization needs further investigations.

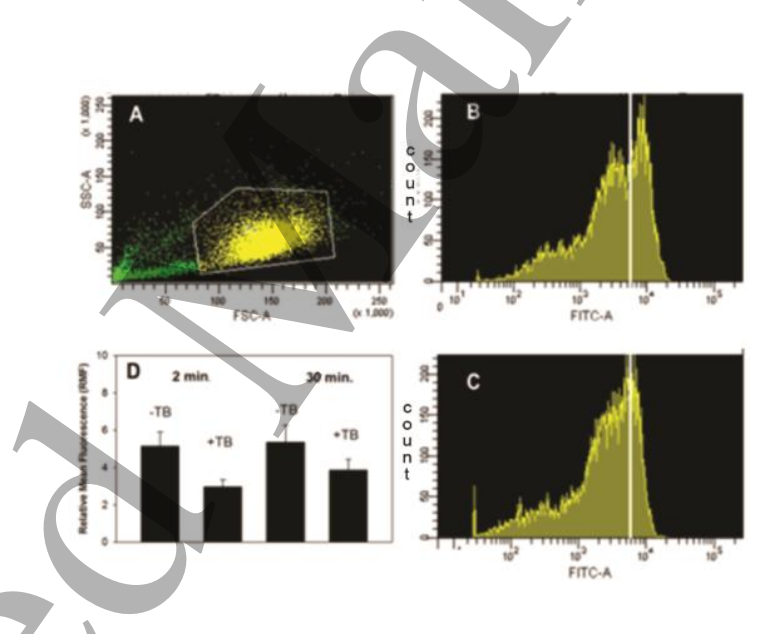

Figure 2. Internalization of surface bound CTX-B as monitored by flow cytofluorimetry.

The forward scatter (FSC) vs. side scatter (SSC) diagram of the B lymphocyte (A20) sample and the gating protocol for selecting live, intact B cells is shown on panel A. Panels $\mathbf{B}$ and $\mathbf{C}$ show representative flow cytometric fluorescence histograms of Alexa488-CTX challenged A20 B lymphocytes in the absence or presence of trypanblue quencher $(0.04 \%)$, respectively. Statistics of flow cytometric experiments performed with or without trypanblue at 2 minutes or 30 minutes after Alexa488-CTX-B administration is shown on $\mathbf{D}$. Each flow cytometric records were made of at least 20.000 cells and the mean and SD values displayed in Fig.2D were determined from 3 independent experiments. (The differences in Fig. 2D are significant at the level of $\mathrm{p}<0.05$ ) 

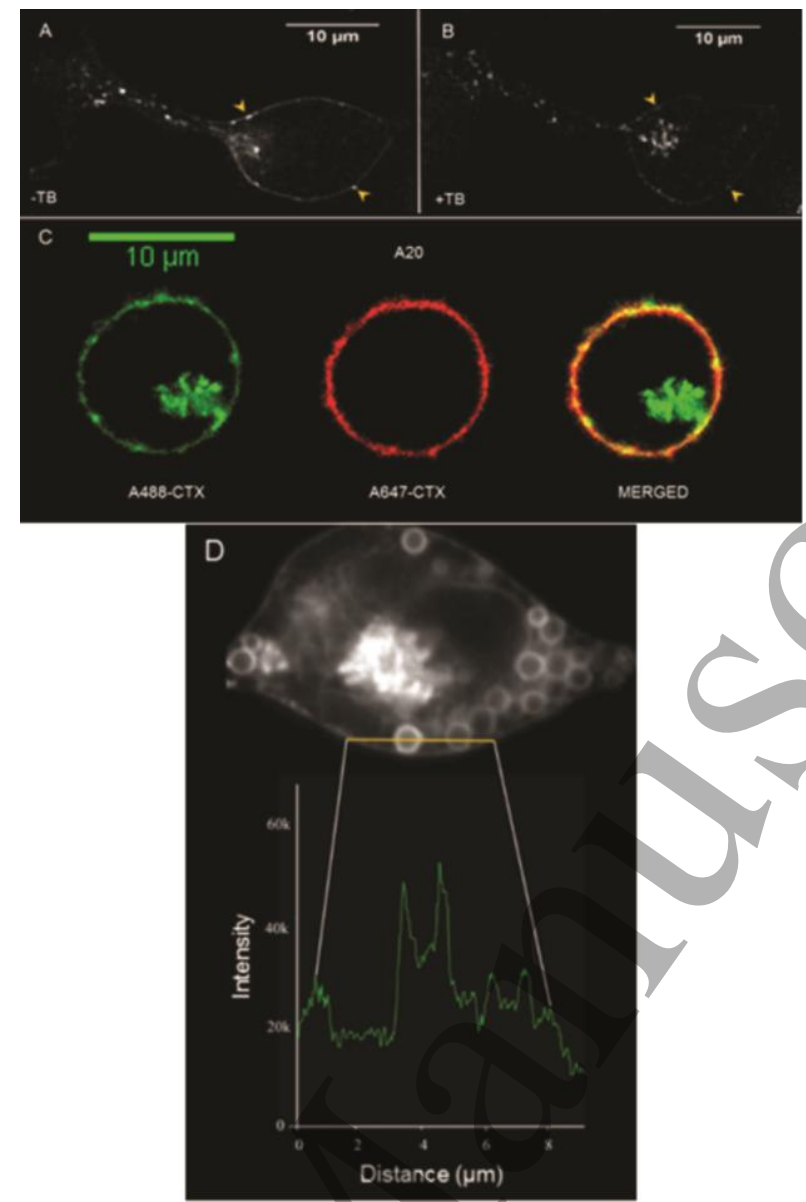

Figure 3. Internalization and properties of CTX-B enriched microvesicles in B cells.

SR-SIM microscopic images of B cells (Fig. 3A and B) clearly show that 30 min after CTX-B challenge addition of trypan-blue $(0.1 \%)$ largely quenched the plasma membrane associated CTX-B fluorescence, while the internal fluorescence was left unchanged. Coexistence of plasma membrane localized and intracellular MV-localized CTX-B fluorescence is shown on Fig. 3C. Fluorescence originated from the cell surface labeling with Alexa488CTX-B (green) is shown 30 min after administration of CTX-B to the B cell culture (left). These cells at this time point were re-stained with Alexa647 (red) (see middle image); merged image of the two is shown right. Panel D shows a representative SR-SIM BW image of a B cell in which the microvesicles are well resolved. A line scan analysis shows the intensity distribution along the scanning line in $\mathrm{X}$ direction. From this analysis the approximate size (diameter) of the individual vesicles can be estimated.

3.3 Intercellular transport of ganglioside-rich MVs between B cells and macrophages: actindependent myosin $2 A$ but not dynein mediates this process

The live cell SR-SIM technology allowed us to record motional trajectories of single microvesicles and thus to analyze their transport properties across tunneling nanotubes bridging lymphocytes and macrophages (Supplementary Movie 1). The Alexa488-CTXstained vesicles were shown to be transported to the neighboring cells (Fig. 4). Existence of such MVs in the intercellular conduits between B cells has already been shown by us (Osteikoetxea-Molnar, Szabo-Meleg et al. 2016), however their motional characteristics have not been analyzed, yet. A two color (green-red; Alexa488 vs. Alexa647), CTX-B labelled mixed culture of B cells was created in order to demonstrate the real intercellular exchange of vesicles. Nanotube formation and the exchange was analyzed by live cell CLSM with 63x magnification (or sometimes with monocolor superresolution SIM imaging). As Fig. 4A shows 
the vesicles formed in a mixed culture of green and red labelled B cells indeed arrive into the 'target cells'. The Supplementary Movie 2 convincingly demonstrates using a superior tracking analysis that a vesicle formed in a B cell (upper; 'donor cell') is transported via the intercellular NT bridge into another B cell (lower; 'acceptor cell'). A single microvesicle is typically transferred in ca. 16 min through the TNT. During a 2 hrs interval in culture, allowing live cell imaging without significant cell death, an actual intercellular exchange of 2-3 microvesicles in average (mean+/-SD: $2+/-2$, from 49 independent fields of view) was observed between the B cells connected with NT conduits. Fig. 4B shows a section of a TNT connecting two B cells (upper part) and this region is zoomed in below (lower part). Fig. 4C is a similar zoomed image showing such moving CTX-B+-microvesicles in a TNT bridging two RAW264.7 macrophage cells.

As shown in Fig. 4D both B and macrophage cells can transport these microvesicles efficiently, since the percentage of mobile vesicles in TNTs were relatively high (ca. 80 and $58 \%$, respectively). Their motion in live cell time-lapse SR-SIM images was analyzed on the basis of their kymographic trajectories and found relatively diverse (Fig. 4E). Their average velocity in the nanotubes was found ca. $15 \pm 3 \mathrm{~nm} / \mathrm{sec}$. Such velocity suggests that the process is likely not free diffusion, but rather mediated by a motor protein-cytoskeletal track system.

Non-muscle myosin 2A (NM2A) motor protein is abundantly expressed in lymphocytes (Osteikoetxea-Molnar, Szabo-Meleg et al. 2016), such as dynein, which is also a functionally essential motor protein in B cells, involved among others in internalization of the antigen receptor, BCR (Schnyder, Castello et al. 2011). So, next we investigated using live cell timelapse SR-SIM imaging, whether inhibition of activities of these motor proteins can affect or not the transport of MVs across TNTs. Inhibition of microtubule associated dynein motor protein activity by ciliobrevin D (up to $20 \mu \mathrm{M}$ concentration) did not affect the transport of MVs, even after 20 minutes incubation (Fig. 4F and Supplementary Movies 1, 3). In the same time window, inhibition of myosin $2 \mathrm{~A}$, an actin-dependent motor protein, by p-nitroblebbistatin (already at $40 \mu \mathrm{M}$ concentration), however, significantly blocked the motion of these vesicles in the tubules (Fig. 4F and Supplementary Movies 1, 4). 

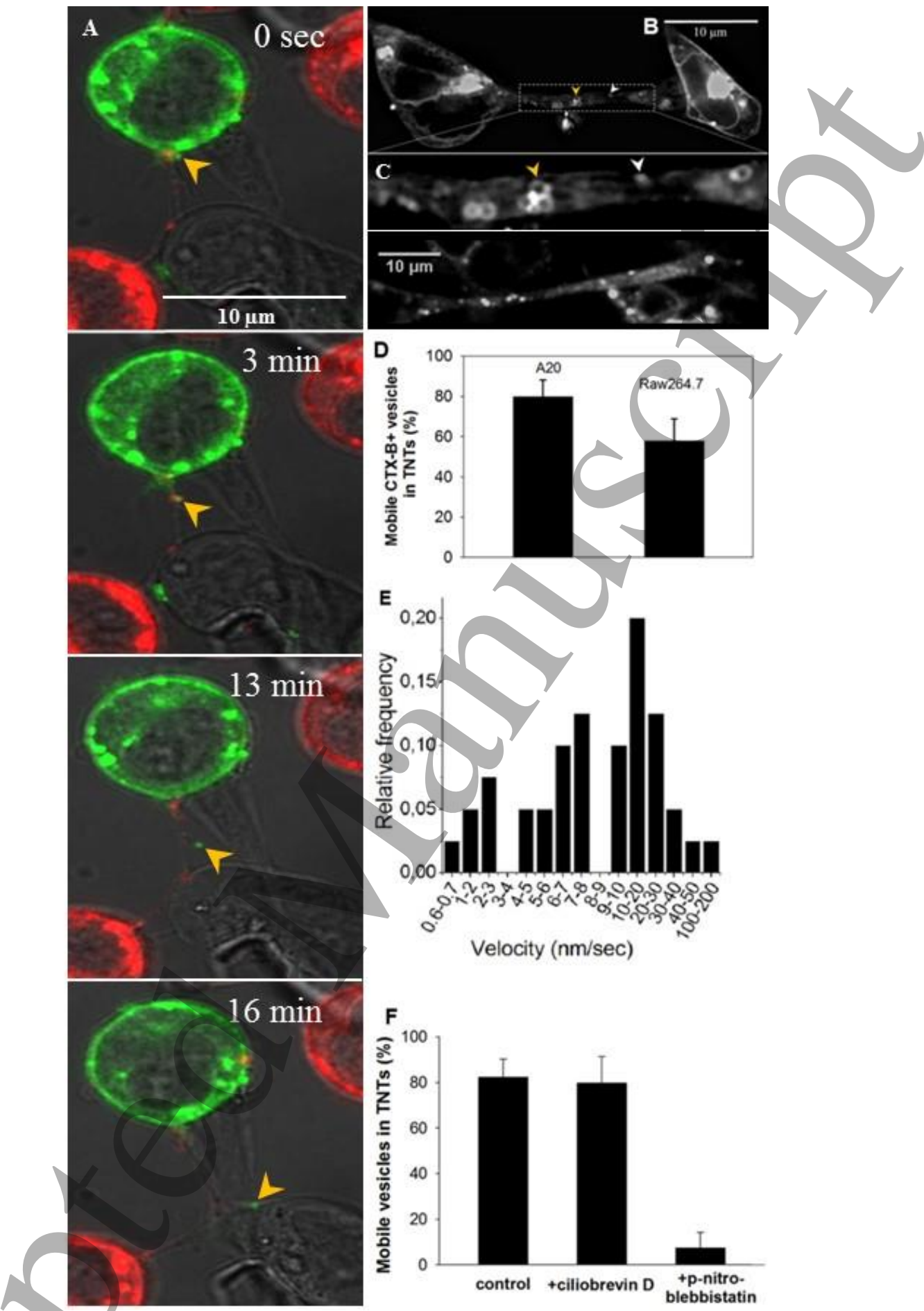

Figure 4. Traffic of ganglioside/CTX enriched microvesicles between B cells and macrophages.

The MVs formed after ganglioside/CTX-B internalization are transported across the TNTs grown under live cell conditions. The time lapse panel (A) is a series of representative CLSM images demonstrating this transport between two adjacent A20 murine B cells (total time: ca.16 min; for the actual position of the moving green vesicle, see the yellow arrowheads). Trajectory of the green-labelled vesicle moving from the donor to the recipient B cell via TNT can be seen in Supplementary Movie 2. Panel B shows zooming into a region of such B cells' TNTs (arrowheads point on individual MVs). A similar representative zoomed image of transported microvesicles along a TNT connecting RAW 264.7 macrophages is shown on panel C. Panel D demonstrates a relatively high mobility of intracellular MVs in both A20 B cells and RAW 264.7 macrophages. As shown in panel $\mathbf{E}$, the mobile vesicles move with a highly diverse velocity, in the range of 0.6 to $200 \mathrm{~nm} / \mathrm{sec}$. Velocity distribution of microvesicles in the tubes was determined by analyzing their kymographic trajectories (FIJI 
software MultipleKymograph plugin). Data showing the percentage of mobile vesicles/TNT revealed that treatment of B cell cultures with selective inhibitor of dynein motor protein (Ciliobrevin-D, at $20 \mu \mathrm{M}$ ) did not influence, but with the inhibitor of actin-based myosin $2 \mathrm{~A}$ motor protein (p-nitro-blebbistatin, at $40 \mu \mathrm{M}$ ) significantly blocked the transport of such MVs (Fig. 4F). The mean +/- SD values of mobile vesicle \% in TNTs determined from three independent experiments (ca. 20-30 TNTs in each samples) are displayed. (These inhibition experiments can also be seen in Supplementary Movies 3-4, with control, untreated cells on Supplementary Movie 1).

\subsection{Transport of B7 family costimulatory molecules between TNT-connected B lymphocyte or macrophage cells}

The actual membrane level of CD86 and CD80 B7-family proteins in B cells and especially in macrophages (where they have typically lower expression) essentially determines the capacity of these cells to activate helper or cytotoxic/killer T cells and the subsequent cellular immune response (Nurieva, Liu et al. 2009). Thus, it is an intriguing question whether these potential antigen-presenting cells can exchange CD86 costimulatory molecules via intercellular transport through nanotubular bridges. Recently we have shown existence of both MHC-IIpeptide complexes and CD86 costimulatory membrane proteins in the nanotubes connecting adjacent B cells (Osteikoetxea-Molnar, Szabo-Meleg et al. 2016).

To investigate redistribution/motion of CD86 costimulatory molecules, in the present study we made fluorescent GFP-CD86 constructs and transfected them into murine B (A20) and macrophage (RAW 264.7) cells. Then, we analyzed spatial distribution of these fluorescent CD86 constructs and the way they are transported between two B or two macrophage cells.

Since both cell types constitutively express endogenous CD86, as well, although at different level (macrophages express significantly less CD86), first we tried to visualize how the transfected green fluorescent CD86 relates spatially to the endogenous CD86 stained by red immunocytochemical (antibody) labeling using permeabilization/fixation of the cells. In B cells (Fig. 5A) the endogenous CD86 (red) shows a more dispersed distribution in the cytoplasm (reflecting also CD86+ intracellular vesicles in which they are transported to the plasma membrane after their synthesis) than in macrophages where their synthesis/expression is much lower (Fig. 5B). In both cells the transfected GFP-CD86 (green) mostly appears in the plasma membrane or in macrophages rarely in large vesicular compartments (Fig. 5A, B and D).

Since it also remained an open question whether in B cells or macrophages these CD86 costimulatory transmembrane proteins can be associated or not with the ganglioside-rich CTX$\mathrm{B}+$-microvesicles mentioned earlier, next we investigated how the transfected green CD86 molecules relate spatially to the red-CTX-B marked ganglioside-rich microvesicles. As Fig. 5C and $\mathbf{D}$ show, there is some level of colocalization between the MVs and the GFP-CD86 (see arrowheads), while some disparate, heavily loaded CD86 compartments (large 'microvesicle-like' structures) could also be observed, but only in macrophages (Fig. 5D). This suggests that in B cells and macrophages the intracellular compartmentation and trafficking of the transfected GFP-CD86 proteins may be largely different. In macrophages we investigated by immunocytochemical/staining of Caveolin-1, a specific structural protein and widely accepted marker of caveolas, whether the transfected CD86 is compartmented in caveolas. As shown in Fig. 5E,F no colocalization is observed between the GFP-CD86 molecules and the red colored antibody labeling caveolin-1, suggesting that the GFP-CD86 complexes are not internalized with Cav-mediated pathways (Pearson colocalization coefficient: $-0,05867+/-$ 0,21603). As a control, Fig. 5G shows that if the endogenous CD86 is labelled extracellularly by antibody (green, in the middle) in B cells (DIC image on left), we see an intensive membrane labeling that can be fully quenched by $0.1 \%$ trypan blue (TB) (right). 
Interestingly enough, the intercellular transport of GFP-CD86 in B cells and macrophages showed a highly different picture. In mature B cells the CD86 transmembrane protein seemed to be transported intercellularly in the membrane of the tunneling nanotubes, rather than in the interior of TNTs (Fig. 5I and Supplementary Movie 5). In contrast, in macrophages, the GFP-CD86 seems to be localized in large 'microvesicle-like' compartments inside the cells, and their intercellular transport also takes place in such elongated (ca. 3-4 $\mu \mathrm{m}$ long and 0.7-0.8 $\mu \mathrm{m}$ thick) structures (Fig. 5H, $\mathbf{J}$ and Supplementary Movie 6). Whatever is the exact mechanism of the transport, our results point out that both B cells and macrophages are able to exchange B7-family costimulatory proteins via nanotubular connections. 

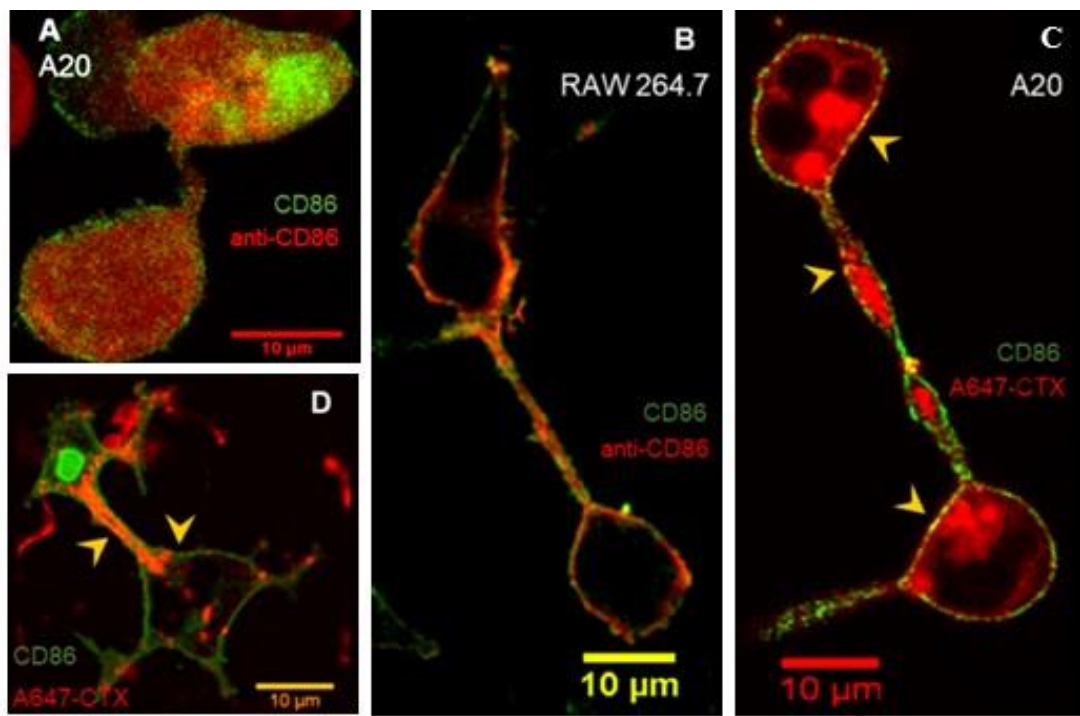

D

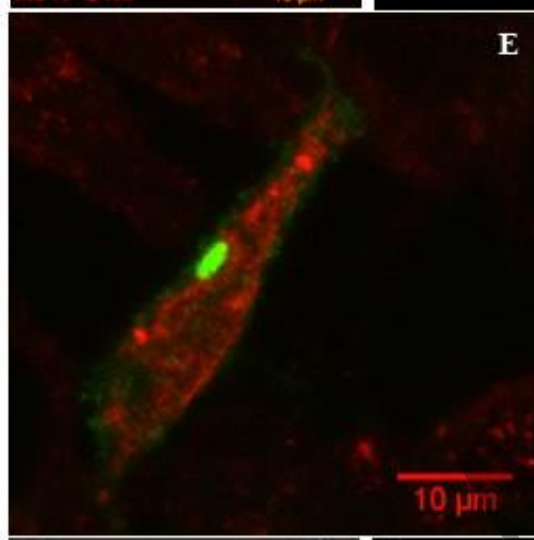

E

$10 \mu \mathrm{m}$
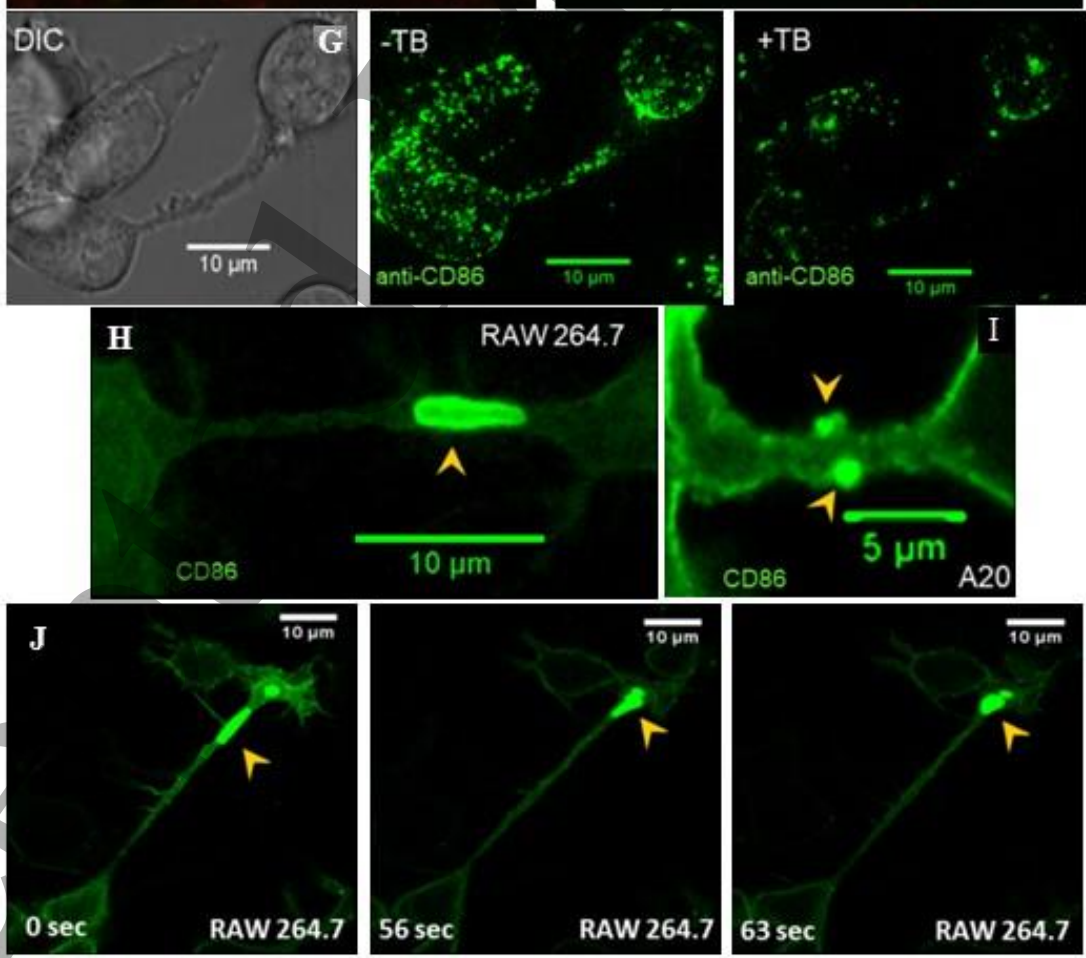

Figure 5. Distribution and intercellular transport of GFP-CD86 protein in macrophages and B cells. GFP-CD86 constructs were transfected into RAW 264.7 macrophage and A20 B cells, respectively. Then cells were visualized under live cell conditions $\left(37{ }^{0} \mathrm{C}, 5 \% \mathrm{CO}_{2}\right.$ atmosphere on $10 \mu \mathrm{g} / \mathrm{ml}$ fibronectin coat) by confocal or SR-SIM microscopy. A: Colocalization of endogenous CD86 (labeled by anti-CD86 antibody+Alexa594secondary Ab; red) with transfected GFP-CD86 (green) in A20 B cells. Panel B demonstrates that colocalization 


\section{DISCUSSION}

Membrane nanotubes (Gerdes, Bukoreshtliev et al. 2007, Davis and Sowinski 2008) and extracellular vesicles (Thery, Ostrowski et al. 2009, Gyorgy, Szabo et al. 2011) are recently recognized novel intercellular communication/transport pathways. Their appearance and potential functional significance were described in many cell types of the immune- and central nervous systems, kidney cells or various tumor cell types (Austefjord, Gerdes et al. 2014). Because of their potential to control cell differentiation, survival, signal transduction, or other secondary cell responses, nanotubular connections gained special attention in the fields of immunology, neurobiology and especially in tumor biology.

Earlier findings from our and other groups (Kabaso, Lokar et al. 2011, Lokar, Kabaso et al. 2012, Osteikoetxea-Molnar, Szabo-Meleg et al. 2016, Toth, Oszvald et al. 2017) showed that formation of nanotubes strongly depends on $\mathrm{GM}_{1} / \mathrm{GM}_{3}$ ganglioside and cholesterol content of the plasma membranes. Our recent model (Toth, Oszvald et al. 2017) proposed that the actual number of plasma membrane lipid rafts, as coupling sites between the plasma membrane and F-actin may be an essential factor determining the number of potentially outgrowing nanotubules. Membrane gangliosides $\mathrm{GM}_{1}$ and $\mathrm{GM}_{3}$, however, are also important in immune cells, as receptors of bacterial toxins, such as various enterotoxins or Cholera toxin (Holmgren and Lindholm 1976, Krengel and Bousquet 2014) with which they may meet frequently. Therefore, it is an intriguing question what happens with the target cells (such as B, T lymphocytes or macrophages) upon engagement of these surface gangliosides by bacterial toxins, such as Cholera toxin. The immature B cells (e.g. the 38C13 cell line) investigated here express much less gangliosides than the mature ones and could not grow nanotubes under the same conditions as the mature B cells did (Toth, Oszvald et al. 2017).

In this study we demonstrate that Cholera toxin B challenge of B cells of immature and mature types leads to formation of small microvesicles through internalization of $\mathrm{GM}_{1} / \mathrm{GM}_{3}$ ganglioside-rich plasma membrane fractions of the cells into their cytoplasm. Internalization starts shortly after the CTX-B treatment and reaches its peaks after approximately 30 minutes. Note that the extent of internalization was different in immature and mature B cells. Immature $\mathrm{B}$ cells form much less MV structures with a lower rate than the mature ones, in accordance with their lower $\mathrm{GM}_{1} / \mathrm{GM}_{3}$ expression level (Toth, Oszvald et al. 2017) in their plasma membràne. However, the exact biological role and the molecular machinery of such MVformation still remained unclear. Nonetheless, our data highlight an important point that only mature B cells can respond to bacterial toxins with signaling (Schnitzler, Burke et al. 2007) and/or intercellular communication/microvesicle-transport. Our data also point out that such 
difference in the plasma membrane lipid composition (in the levels of gangliosides or in other lipid species promoting proper membrane curvature; as reported by Toth EA et al. 2017) may affect not only the extent of nanotube formation, but also the subsequent microvesicle formation, after internalization.

Live cell SR-SIM superresolution imaging (Vangindertael, Camacho et al. 2018) technique with its improved lateral resolution $(\mathrm{d}: \sim 80-90 \mathrm{~nm})$ provided valuable novel information about the characteristic properties (e.g. morphology, size- and velocitydistribution) of these MVs and about their transport through the TNTs connecting these immune cells. We demonstrated here that SR-SIM can sufficiently monitor the intercellular transport of these microvesicles, using both tracking and kymographic analysis of their motional trajectories. Analyzing their tracks and velocity distribution in the TNTs, we found that they move bidirectionally with a relatively low velocity $(15-20 \mathrm{~nm} / \mathrm{s}$, in average). Such velocities suggest that they may be transported as cargos mediated by actin- or microtubuleassociated motor protein activities. Our data strongly suggest that the dominant motor protein mediating this transport, is the actin-dependent non-muscle myosin $2 \mathrm{~A}$ in these cells. According to our results, the other abundant, microtubule-associated motor protein in B cells, dynein, was not involved in their transfer. This observation highlights the possibility of modulating such nanotubular intercellular transport e.g. by specific targeting of a motor protein to inhibit its cargo-transport. The low number of MVs transported intercellularly between B cells is likely due to the fact that adjacent B cells are often connected by more than one nanotube, and these can be either thin (with closed end) or thick (open ended, or tunneling) NTs (Osteikoetxea-Molnar et al, 2016, Cell MolLife Sci.). In contrast to the cell tracking dyes (DiI, DiD or DiO), staining the NTs basically uniformly, the FL-CTX-B challenge induces vesiculation and internalization after binding to cell surface gangliosides, regardless of the color of conjugated dye to CTX-B, and many of the vesicle-cargos formed this way may not reach the target cells because of the closed end of some NTs.

Beyond being a 'passive' platform of hijacking molecules, prions, viruses or for surfing bacteria, membrane nanotubes may also serve as active 'communication channels'. It was e.g. demonstrated that immune cells can exchange protein (or lipid) molecules through the process called 'trogocytosis' that can on the other hand affect many stages of the immune response, e.g. effector responses of/lymphocytes, cell killing, cell death/homeostasis, etc. (Onfelt, Nedvetzki et al. 2004, Davis and Sowinski 2008, Davis 2009).

The intercellular transport pathways shown here arise an important question: what might be the biological significance of the molecular transport across TNTs between the same or different cell types? So far, we know that the cells may inform each other about lack of energy (compensated e.g. by transfer of mitochondria) or tumor cells may transfer even resistance factors to each other via NTs (Osswald, Jung et al. 2015). Immune cells may transfer this way to each other molecules in the TNT membrane or inside the tube, packed in microvesicles, that may modulate (either activate or inhibit) different steps of the adaptive immune response (Thery, Ostrowski et al. 2009, Gyorgy, Szabo et al. 2011). Such intercellular transport processes described in the present study may result in the so called "cross-dressing" of the connected cells (Dhainaut and Moser 2014, Campana, De Pasquale et al. 2015, Zaccard, Watkins et al. 2015, Zaccard, Rinaldo et al. 2016), especially of antigen presenting cells (APCs), which may lead to a more efficient antigen presentation and $\mathrm{T}$ cell activation, important for an efficient cellular immune response.

Our results also pointed on the differences in intracellular compartmentation, traffic and intercellular transport via TNTs of the GFP-CD86 costimulatory protein in B cells vs. macrophages. In B cells these proteins are transported dominantly along the membrane of the TNTs, while in macrophages the transfected GFP-CD86 appears to form special intracellular compartments, in highly dense, large 'microvesicle-like' structures and intercellularly 
transported in these unusual, elongated structures inside the TNTs. We do not know yet what is the reason of this difference, but it is likely due to the different internalization pathways, and intracellular protein storage/compartmentation mechanisms in macrophages and B cells, respectively (see e.g. the unusually high density of CD86 in the 'microvesicle-like' structures in macrophages) (Biswas, Biswas et al. 2009, Xu, Barnes et al. 2010). A dynamic intracellular CD86 reservoir described and characterized earlier (Smyth, Logan et al. 1998, Smyth, Logan et al. 2005) showing a cell-type specific subcellular compartmentation of CD86 seems to support our findings regarding the differences in the transportation mechnisms.

Regardless of the exact mechanism, which obviously needs further examination, the presented work explored that both B cells and macrophages (as potential antigen presenting cells) can exchange CD86, a centrally important costimulator in $\mathrm{T}$ cell activation, via nanotubular connections. Our findings helped to reveal new aspects of intercellular transport of immunoregulatory factors and hence to recognize novel potential immunomodulation pathways. Exact identification of all the significant immunoregulatory components carried by the ganglioside-rich, CTX-B+ microvesicles described here, of course is another important task, which needs further detailed investigations. Our results otherwise also highlighted the power of SR-SIM (or other) superresolution imaging techniques (Vangindertael, Camacho et al. 2018) over live cell confocal microscopy in revealing 'submicroscopic' structural or kinetic details of novel biological transport pathway, such as mícrovesicle transport via intercellular nanotubular networks. 


\section{Acknowledgements}

This work was supported by grants K104971, NN 107776 and K112794 from National Research Development and Innovation Office (NKFIH). This work was also supported by the ÚNKP-17-4-IV New National Excellence Program of the Ministry of Human Capacities (to SzME), the GINOP-2.3.2-15-2016-00036, and the EFOP-3.6.1-16-2016-00004. ZB is a Bolyai Research Fellow. The authors would like to thank to Nóra Henn-Mike for her assistance in endogenous CD86 labeling and to Dr. Glória László for the helpful advice in works with CD86 and the continuous valuable discussions.

The present scientific contribution is dedicated to the 650th anniversary of the foundation of the University of Pécs, Hungary.

\section{References}

Ady, J., V. Thayanithy, K. Mojica, P. Wong, J. Carson, P. Rao, Y. Fong and E. Lou (2016). "Tunneling nanotubes: an alternate route for propagation of the bystander effect following oncolytic viral infection." Mol Ther Oncolytics 3: 16029.

Austefjord, M. W., H. H. Gerdes and X. Wang (2014). "Tunneling nanotubes: Diversity in morphology and structure." Commun Integr Biol 7(1): e27934.

Biswas, S., K. Biswas, A. Richmond, J. Ko, S. Ghosh, M. Simmons, P. Rayman, B. Rini, I. Gill, C. S. Tannenbaum and J. H. Finke (2009). "Elevated levels of select gangliosides in T cells from renal cell carcinoma patients is associated with $\mathrm{T}$ cell dysfunction." $\mathrm{J}$ Immunol 183(8): 5050-5058.

Campana, S., C. De Pasquale, P. Carrega, G. Ferlazzo and I. Bonaccorsi (2015). "Crossdressing: an alternative mechanism for antigen presentation." Immunol Lett 168(2): 349-354. Caspi, Y., M. Taya, N. Hollander and J. Haimovich (1995). "Light chain loss and reexpression leads to idiotype switch. Surrogate light chains are probably responsible for this process." Curr Top Microbiol Immunol 194: 179-186.

Davis, D. M. (2009). "Mechanisms and functions for the duration of intercellular contacts made by lymphocytes." Nat Rev Immunol 9(8): 543-555.

Davis, D. M. and S. Sowinski (2008). "Membrane nanotubes: dynamic long-distance connections between animal cells." Nat Rev Mol Cell Biol 9(6): 431-436.

Delage, E. and C. Zurzolo (2013). "Exploring the role of lipids in intercellular conduits: breakthroughs in the pipeline." Front Plant Sci 4: 504.

Dhainaut, M. and M. Moser (2014). "Regulation of immune reactivity by intercellular transfer." Front Immunol 5: 112.

Gerdes, H. H., N. V. Bukoreshtliev and J. F. Barroso (2007). "Tunneling nanotubes: a new route for the exchange of components between animal cells." FEBS Lett 581(11): 2194-2201. Gerdes, H. H, and R. N. Carvalho (2008). "Intercellular transfer mediated by tunneling nanotubes." Curr Opin Cell Biol 20(4): 470-475.

Gurke, S., J. F. Barroso and H. H. Gerdes (2008). "The art of cellular communication: tunneling nanotubes bridge the divide." Histochem Cell Biol 129(5): 539-550.

Gyorgy, B., T. G. Szabo, M. Pasztoi, Z. Pal, P. Misjak, B. Aradi, V. Laszlo, E. Pallinger, E. Pap, A. Kittel, G. Nagy, A. Falus and E. I. Buzas (2011). "Membrane vesicles, current stateof-the-art: emerging role of extracellular vesicles." Cell Mol Life Sci 68(16): 2667-2688.

Holmgren, J. and L. Lindholm (1976). "Cholera toxin, ganglioside receptors and the immune response." Immunol Commun 5(9): 737-756.

Janakiram, M., Y. M. Abadi, J. A. Sparano and X. Zang (2012). "T cell coinhibition and immunotherapy in human breast cancer." Discov Med 14(77): 229-236. 
Kabaso, D., M. Lokar, V. Kralj-Iglic, P. Veranic and A. Iglic (2011). "Temperature and cholera toxin B are factors that influence formation of membrane nanotubes in RT4 and T24 urothelial cancer cell lines." Int J Nanomedicine 6: 495-509.

Kepiro, M., B. H. Varkuti, L. Vegner, G. Voros, G. Hegyi, M. Varga and A. MalnasiCsizmadia (2014). "para-Nitroblebbistatin, the non-cytotoxic and photostable myosin II inhibitor." Angew Chem Int Ed Engl 53(31): 8211-8215.

Krengel, U. and P. A. Bousquet (2014). "Molecular recognition of gangliosides and their potential for cancer immunotherapies." Front Immunol 5: 325.

Lokar, M., D. Kabaso, N. Resnik, K. Sepcic, V. Kralj-Iglic, P. Veranic, R. Zorec and A. Iglic (2012). "The role of cholesterol-sphingomyelin membrane nanodomains in the stability of intercellular membrane nanotubes." Int J Nanomedicine 7: 1891-1902.

Lou, E. (2016). "Intercellular Conduits in Tumors: The New Social Network." Trends Cancer 2(1): 3-5.

Lou, E., S. Fujisawa, A. Barlas, Y. Romin, K. Manova-Todorova, M. A. Moore and S. Subramanian (2012). "Tunneling Nanotubes: A new paradigm for studying intercellular communication and therapeutics in cancer." Commun Integr Biol 5(4): 399-403.

Nurieva, R. I., X. Liu and C. Dong (2009). "Yin-Yang of costimulation: crucial controls of immune tolerance and function." Immunol Rev 229(1): 88-100.

Onfelt, B., S. Nedvetzki, R. K. Benninger, M. A. Purbhoo, S. Sowinski, A. N. Hume, M. C. Seabra, M. A. Neil, P. M. French and D. M. Davis (2006). "Structurally distinct membrane nanotubes between human macrophages support long-distance vesicular traffic or surfing of bacteria." J Immunol 177(12): 8476-8483.

Onfelt, B., S. Nedvetzki, K. Yanagi and D. M. Davis (2004). "Cutting edge: Membrane nanotubes connect immune cells." J Immúnol 173(3): 1511-1513.

Osswald, M., E. Jung, F. Sahm, G. Solecki, V. Venkataramani, J. Blaes, S. Weil, H. Horstmann, B. Wiestler, M. Syed, L. Huang, M. Ratliff, K. Karimian Jazi, F. T. Kurz, T. Schmenger, D. Lemke, M. Gommel, M. Pauli, Y. Liao, P. Haring, S. Pusch, V. Herl, C. Steinhauser, D. Krunic, M. Jarahian, H. Miletic, A. S. Berghoff, O. Griesbeck, G. Kalamakis, O. Garaschuk, M. Preusser, S. Weiss, H. Liu, S. Heiland, M. Platten, P. E. Huber, T. Kuner, A. von Deimling, W. Wick and F. Winkler (2015). "Brain tumour cells interconnect to a functional and resistant network." Nature 528(7580): 93-98.

Osteikoetxea-Molnar, A., E. Szabo-Meleg, E. A. Toth, A. Oszvald, E. Izsepi, M. Kremlitzka, B. Biri, L. Nyitray, T. Bozo, P. Nemeth, M. Kellermayer, M. Nyitrai and J. Matko (2016). "The growth determinants and transport properties of tunneling nanotube networks between B lymphocytes." Cell Mol Life Sci 73(23): 4531-4545.

Patino, T., J. Soriano, L. Barrios, E. Ibanez and C. Nogues (2015). "Surface modification of microparticles causes differential uptake responses in normal and tumoral human breast epithelial cells." Sci Rep 5: 11371.

Rustom, A., R. Saffrich, I. Markovic, P. Walther and H. H. Gerdes (2004). "Nanotubular highways for intercellular organelle transport." Science 303(5660): 1007-1010.

Schermelleh, L., R. Heintzmann and H. Leonhardt (2010). "A guide to super-resolution fluorescence microscopy." J Cell Biol 190(2): 165-175.

Schnitzler, A. C., J. M. Burke and L. M. Wetzler (2007). "Induction of cell signaling events by the cholera toxin B subunit in antigen-presenting cells." Infect Immun 75(6): 3150-3159.

Schnyder, T., A. Castello, C. Feest, N. E. Harwood, T. Oellerich, H. Urlaub, M. Engelke, J. Wienands, A. Bruckbauer and F. D. Batista (2011). "B cell receptor-mediated antigen gathering requires ubiquitin ligase $\mathrm{Cbl}$ and adaptors Grb2 and Dok-3 to recruit dynein to the signaling microcluster." Immunity 34(6): 905-918.

Sherer, N. M. and W. Mothes (2008). "Cytonemes and tunneling nanotubules in cell-cell communication and viral pathogenesis." Trends Cell Biol 18(9): 414-420. 
Smyth, C., G. Logan, R. P. Weinberger, P. B. Rowe, I. E. Alexander and J. A. Smythe (1998). "Identification of a dynamic intracellular reservoir of CD86 protein in peripheral blood monocytes that is not associated with the Golgi complex." J Immunol 160(11): 5390-5396.

Smyth, C. M., G. Logan, R. Boadle, P. B. Rowe, J. A. Smythe and I. E. Alexander (2005). "Differential subcellular localization of CD86 in human PBMC-derived macrophages and DCs, and ultrastructural characterization by immuno-electron microscopy." Int Immunol 17(2): 123-132.

Thery, C., M. Ostrowski and E. Segura (2009). "Membrane vesicles as conveyors of immune responses." Nat Rev Immunol 9(8): 581-593.

Toth, E. A., A. Oszvald, M. Peter, G. Balogh, A. Osteikoetxea-Molnar, T. Bozo, E. SzaboMeleg, M. Nyitrai, I. Derenyi, M. Kellermayer, T. Yamaji, K. Hanada, L. Vigh and J. Matko (2017). "Nanotubes connecting B lymphocytes: High impact of differentiation-dependent lipid composition on their growth and mechanics." Biochim Biophys Acta 1862(9): 991-1000.

Vangindertael, J., R. Camacho, W. Sempels, H. Mizuno, P. Dedecker and K. P. F. Janssen (2018). "An introduction to optical super-resolution microscopy for the adventurous biologist." Methods Appl Fluoresc 6(2): 022003.

$\mathrm{Xu}$, Y. H., S. Barnes, Y. Sun and G. A. Grabowski (2010). "Multi-system disorders of glycosphingolipid and ganglioside metabolism." J Lipid Res 51(7): 1643-1675.

Zaccard, C. R., C. R. Rinaldo and R. B. Mailliard (2016). "Linked in: immunologic membrane nanotube networks." J Leukoc Biol 100(1): 81-94.

Zaccard, C. R., S. C. Watkins, P. Kalinski, R. J. Fecek, A. L. Yâtes, R. D. Salter, V. Ayyavoo, C. R. Rinaldo and R. B. Mailliard (2015). "CD40L induces functional tunneling nanotube networks exclusively in dendritic cells programmed by mediators of type 1 immunity." $\underline{\mathbf{J}}$ Immunol 194(3): 1047-1056. 